\title{
VOLTAMETRIA DE ONDA QUADRADA. SEGUNDA PARTE: APLICAÇÕES
}

\author{
Djenaine de Souza*, Lúcia Codognoto, Andréa R. Malagutti, Renata A. Toledo, Valber A. Pedrosa, Robson T. S. Oliveira, \\ Luiz H. Mazo, Luis A. Avaca e Sergio A. S. Machado
}

Instituto de Química de São Carlos, Universidade de São Paulo, CP 780, 13560-970 São Carlos - SP

Recebido em 3/10/03; aceito em 14/1/04; publicado na web em 17/6/04

\begin{abstract}
SQUARE WAVE VOLTAMMETRY. SECOND PART: APPLICATIONS. The aim of this work is to discuss some selected applications of square wave voltammetry published in the last five years. The applications focused here cover several electroanalytical fields such as: determination of pesticides; molecules with biological activity; metals and other environmental pollutants. Special attention is given to the work developed in the Grupo de Materiais Eletroquímicos e Métodos Eletroanalíticos - IQSC - USP concerning the utilization of square wave voltammetry, with different kinds of electrodes, for the determination of pesticides in natural waters and active principles in pharmaceutical formulations. The new methodology is simple, fast and sensitive when compared with the traditional ones such as chromatography and spectrophotometry. The satisfactory results obtained provide alternative procedures for the quality control of drugs and the monitoring of pesticides in natural environments.
\end{abstract}

Keywords: square wave voltammetry; electroanalysis; electrochemical determinations.

\section{INTRODUÇÃO}

A voltametria de onda quadrada ("SWV - square wave voltammetry") é uma técnica voltamétrica de pulso onde a forma do pico de corrente resultante é proveniente da sobreposição de pulsos de potencial de altura $a$ (amplitude de pulsos), a uma escada de potenciais de largura $\Delta E_{s}$ (incremento de varredura de potenciais) e duração $2 t$ (período). As medidas de corrente são feitas no final dos pulsos diretos e reversos e o sinal obtido, após derivação, é dado como uma intensidade da corrente resultante, apresentando excelente sensibilidade e alta rejeição a correntes capacitivas ${ }^{1}$. O pico voltamétrico resultante apresenta posição, largura e altura características do tipo de sistema redox avaliado ${ }^{2}$. A Figura 1 apresenta a forma de aplicação do potencial da SWV.

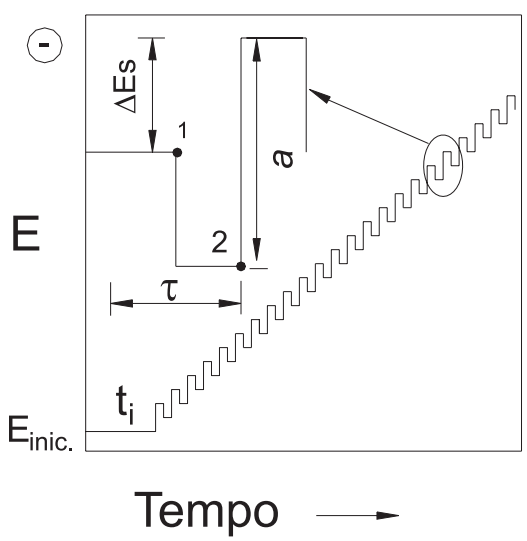

Figura 1. Forma de aplicação do potencial na voltametria de onda quadrada

Atualmente, a SWV é uma das técnicas de pulso mais rápidas e sensíveis entre todas as outras. Os limites de detecção obtidos com

*e-mail: djenaine@iqsc.usp.br sua utilização podem ser comparados àqueles das técnicas cromatográficas e espectroscópicas.

Uma das grandes vantagens da SWV em relação às outras técnicas voltamétricas é que sua teoria e os modelos matemáticos utilizados nos critérios de diagnóstico de tipos de processos redox foram desenvolvidos considerando-se, também, as espécies eletroativas adsorvidas na superfície eletródica e reações redox totalmente irreversíveis. Tais critérios de diagnóstico são muito importantes em eletroquímica, especialmente na análise de compostos orgânicos, onde os processos de adsorção provocam um efeito complexo nas respostas voltamétricas, efeito este dependente da quantidade em que reagente, produto, ou ambas as espécies adsorvem, da força e da dependência do potencial de adsorção, do eletrólito suporte utilizado e, também, da forma de aplicação do potencial. Além disto, processos redox totalmente irreversíveis são muito comuns em diversos tipos de compostos orgânicos contendo anéis aromáticos homocíclicos ou heterocíclicos com grupos ligantes eletroativos. Tais grupos são superfícies ativas e sofrem reduções e/ou oxidações totalmente irreversíveis, devido à baixa velocidade de transferência de carga, ou, muitas vezes, por uma rápida desativação do produto de reação redox, devido a um processo de protonação irreversível seguido por uma transferência de carga ${ }^{3}$.

Dentre outras importantes vantagens apresentadas pela SWV podem-se destacar:

1) ausência da interferência proveniente da corrente capacitiva, já que as medidas de corrente são realizadas ao final do degrau de potencial onde a corrente capacitiva já se tornou negligenciável, melhorando a resolução dos dados experimentais, a sensibilidade analítica e, ainda, possibilitando a realização das análises em altas velocidades de varredura;

2) a análise de componentes de corrente direta e reversa possibilita a obtenção de dados mecanísticos semelhantes àqueles obtidos por voltametria cíclica, porém com maior sensibilidade, devido à minimização das correntes capacitivas.

Como limitação apresentada pela SWV é importante salientar que, apesar dos modelos teóricos desenvolvidos por Lóvric e Osteryoung abrangerem processos redox reversíveis, irreversíveis e quase-reversíveis, com adsorção de produtos e/ou reagentes e também na ausência 
de processos de adsorção, a aplicação em processos redox envolvendo reações químicas acopladas, ou seja, reações químicas ocorrendo antes ou após a transferência eletrônica ainda se necessitam estudos mais detalhados, a fim de melhor aplicar-se os modelos para estudos mecanísticos e cinéticos. Porém, para aplicações analíticas esta ainda é a técnica voltamétrica que fornece os melhores resultados em termos de intensidade de corrente e perfil voltamétrico, independentemente do tipo de processo redox envolvido.

$\mathrm{Na}$ primeira parte deste trabalho ${ }^{3}$ foi apresentada uma discussão acerca do desenvolvimento da SWV e a formulação dos modelos teóricos aplicados a sistemas reversíveis, irreversíveis e quase-reversíveis. Os avanços recentes na teoria básica e na aplicação de eletrodos convencionais, ultramicroeletrodos, eletrodos hidrodinâmicos e contribuições da múltipla voltametria de onda quadrada também foram avaliados.

Desta forma, em continuidade, o presente trabalho apresenta uma discussão sobre os avanços da SWV para a aplicação em eletroanalítica e no estudo de diferentes processos redox. Uma atenção especial será dada a alguns trabalhos desenvolvidos no Grupo de Materiais Eletroquímicos e Métodos Eletroanalíticos (GMEMEUSP), que utilizam a SWV no estudo de processos redox de vários pesticidas e fármacos, utilizando diferentes superfícies eletródicas.

\section{APLICAÇÕES}

O intenso desenvolvimento tecnológico ocorrido nos últimos anos, que tornou possível a construção de equipamentos com maiores recursos eletrônicos, possibilitou que a SWV se tornasse uma das técnicas de pulso mais empregadas na determinação analítica de compostos orgânicos e inorgânicos, pois é capaz de fornecer resultados tão sensíveis quanto aqueles obtidos nas técnicas cromatográficas ou espectroscópicas, com a vantagem da amostra não necessitar de nenhum tratamento ou separação prévia, o que minimiza o tempo e os gastos despendidos com as análises.

Uma revisão da literatura realizada a partir de 1995 mostra o intenso crescimento na utilização da SWV para fins analíticos. Podese notar uma grande variedade de aplicação da SWV para os mais diversos compostos e em diferentes condições voltamétricas.

Nos últimos anos, a utilização de técnicas eletroanalíticas, e especialmente a SWV, para a determinação de diferentes classes de pesticidas em diferentes amostras tem mostrado crescimento exponencial. Na prática, o que se pode observar na maior parte dos trabalhos relacionados à análise de pesticidas e ao emprego da SWV é que, no estudo dos parâmetros cinéticos e mecanísticos destas moléculas, quase sempre é utilizada a técnica tradicional de voltametria cíclica, enquanto que a SWV fica restrita apenas às determinações analíticas. Isto ocorre porque a teoria envolvida nos modelos de processos eletródicos para a SWV é mais complexa.

Adicionalmente, a intensa utilização das técnicas voltamétricas na análise de compostos com aplicações farmacológicas e de moléculas de interesse biológico está intimamente relacionada com o desenvolvimento de métodos mais sensíveis, que possibilitam a determinação em nível de ultra-traços, principalmente em fluidos biológicos, no controle de qualidade em produtos farmacêuticos e em alimentos. Desta forma, a SWV tem sido amplamente empregada na determinação de tais compostos, apresentando as vantagens da não necessidade de etapas de pré-tratamento de amostras e rapidez de análise em relação às técnicas cromatográficas, o que justifica o aumento de trabalhos, na literatura, empregando essa técnica

A SWV tem se mostrado, também, uma técnica muito conveniente para a análise de metais, seja na determinação de suas respostas eletroquímicas diretas, como modelos experimentais ${ }^{4}$, seja na formação de complexos com outras substâncias de interesse, como as metaloproteínas ${ }^{5}$ ou, ainda, como contaminantes em resíduos industriais $^{6}$. Assim, a SWV é utilizada no estudo de mecanismos de formação de complexos metálicos onde seus critérios de diagnóstico possibilitam a avaliação da estequiometria da reação de formação de complexos, da constante de velocidade da formação de complexos, da influência de processos de adsorção-dessorção de ligantes, além dos efeitos de mudanças de concentração de ligantes ${ }^{7,8}$. A caracterização de eletrodos quimicamente modificados com diferentes complexos tem sido freqüientemente realizada utilizando-se a $\mathrm{SWV}^{9,10}$. Vale destacar que a utilização de complexos na modificação de eletrodos é de grande importância, pois eles catalisam o processo redox de interesse, promovendo uma intensa melhoria na sensibilidade da técnica analítica.

A Tabela 1 apresenta um resumo de alguns trabalhos onde a SWV é utilizada na análise de fármacos, compostos de interesse biológico, pesticidas, determinação de metais e estudos de formação de complexos.

\section{TRABALHOS DESENVOLVIDOS PELO GMEME}

O GMEME-USP vem desenvolvendo, nos últimos anos, uma série de trabalhos visando o desenvolvimento de metodologias eletroanalíticas mais acessíveis para a determinação de compostos de interesse biológico e farmacológico. Como parte da elaboração de uma metodologia mais adequada, os estudos desenvolvidos no GMEME apresentam uma avaliação crítica e detalhada dos mecanismos redox envolvidos. Desta forma, pretende-se contribuir significativamente para o desenvolvimento da SWV como uma ferramenta versátil nos estudos de mecanismos complexos, como aqueles relacionados com as moléculas orgânicas de interesse (pesticidas e fármacos).

A seguir, é apresentada discussão dos principais trabalhos desenvolvidos no GMEME utilizando-se a SWV, aliada a diferentes materiais eletródicos, para a determinação analítica de vários pesticidas, dentre eles picloram, diclorvos, paraquat, 4-clorofenol e pentaclorofenol, e dos fármacos ranitidina e dipiridamol.

\section{Determinação de pesticidas}

\section{Picloram}

O picloram (4-amino-3,5,6-tricloropiridina-2-ácido carboxílico) é um herbicida utilizado no combate a ervas daninhas de folhas largas nas culturas de cana-de-açúcar, milho, sorgo, dentre outras ${ }^{89}$. Para sua determinação eletroanalítica, um estudo eletroquímico prévio foi realizado, utilizando-se o eletrodo de mercúrio (HMDE) em meio de $\mathrm{H}_{2} \mathrm{SO}_{4}(\mathrm{pH} 1,0)$. Nestas condições, a molécula de picloram apresentou dois picos de redução totalmente irreversíveis, o principal em - 0,716 V e um secundário em - 0,774 V vs Ag/AgCl, com os reagentes fortemente adsorvidos ${ }^{81}$. A dependência do potencial de pico de redução com a concentração hidrogeniônica da solução mostrou que este processo eletródico foi precedido por uma protonação prévia da molécula em estudo, e a aplicação dos critérios de diagnóstico da SWV possibilitou a caracterização de uma transferência eletrônica envolvendo 4 elétrons. Estudos posteriores possibilitaram associar estes elétrons à redução de um cloreto (dois elétrons) e à redução do grupo ácido (para o aldeído correspondente).

A aplicação da SWV para determinação analítica do picloram em águas naturais foi realizada por Massaropi et al. ${ }^{81}$. O limite de detecção obtido para água Milli-Q foi $11 \mu \mathrm{g} \mathrm{L}^{-1}(11 \mathrm{ppb})$ enquanto que, para águas poluídas coletadas em riachos da cidade de São Carlos, o limite de detecção ficou entre 11 e $39 \mu \mathrm{g} \mathrm{L}^{-1}$. Estes valores são satisfatórios, já que a Agência de Proteção Ambiental Americana (EPA) define o limite máximo aceitável de picloram em água potável em $0,5 \mathrm{mgL}^{-1}$ (500 ppb). 
Tabela 1. Resumo das aplicações da SWV na determinação eletroanalítica de diferentes compostos

\begin{tabular}{|c|c|c|c|}
\hline Composto & Matriz & LD & Ref. \\
\hline Adenina e Guanina & DNA & $3,7 \mu \mathrm{g} \mathrm{L}^{-1}$ e $10,0 \mu \mathrm{g} \mathrm{L}^{-1}$ & 11 \\
\hline Proteínas & Milho transgênico & $6,0 \times 10^{-12} \mathrm{~mol} \mathrm{~L}^{-1}$ & 12 \\
\hline Cifonicid (antibiótico) & Urina & $1,0 \mathrm{mg} \mathrm{L}^{-1}$ & 13 \\
\hline Noradrenalina (fluido biológico) & Injeção & $7,0 \times 10^{-7} \mathrm{~mol} \mathrm{~L}^{-1}$ & 14 \\
\hline Nifedipina (hipertensivo) & Sangue & $3,9 \times 10^{-10} \mathrm{~mol} \mathrm{~L}^{-1}$ & 15 \\
\hline Dopamina (neurotransmissor) & Injeção & $4,0 \times 10^{-7} \mathrm{~mol} \mathrm{~L}^{-1}$ & 16,17 \\
\hline Sertralina (antidepressivo) & Formulação farmacêutica & $1,9 \times 10^{-7} \mathrm{~mol} \mathrm{~L}^{-1}$ & 18 \\
\hline Serotonina (neurotransmissor) & Sangue, urina & $2 \times 10^{-9} \mathrm{~mol} \mathrm{~L}^{-1} ; 0,8 \mu \mathrm{g} \mathrm{L}^{-1}$ & 19,20 \\
\hline Lansoprazole (antiácido) & Sangue & $0,25 \times 10^{-9} \mathrm{~mol} \mathrm{~L}^{-1}$ & 21 \\
\hline Sulfametazina (antibiótico) & Veterinária & $3,7 \times 10^{-7} \mathrm{~mol} \mathrm{~L}^{-1}$ & 22 \\
\hline Ketorolac (antiinflamatório) & Soro humano & $1,0 \times 10^{-11} \mathrm{~mol} \mathrm{~L}^{-1}$ & 23 \\
\hline Etodolac & Soro humano e formulação farmacêutica & $1,1 \times 10^{-6} \mathrm{~mol} \mathrm{~L}^{-1}$ & 24 \\
\hline Olsalazina & Cápsula & $2,0 \times 10^{-7} \mathrm{~mol} \mathrm{~L}^{-1}$ & 25 \\
\hline Levoflaxin (antibiótico) & Urina & $5,0 \times 10^{-9} \mathrm{~mol} \mathrm{~L}^{-1}$ & 26 \\
\hline Formoterol (broncodilatador) & Cápsula e soro humano & $8,0 \times 10^{-6} \mathrm{~mol} \mathrm{~L}^{-1}$ & 27 \\
\hline $\begin{array}{l}\text { Fluvastatin sódio } \\
\text { (redução de colesterol) }\end{array}$ & Drogas e fluidos biológicos & $1,0 \times 10^{-6} \mathrm{~mol} \mathrm{~L}^{-1}$ & 28 \\
\hline Alfuzosin (anti-cancer) & Sangue e suco gástrico & $6,2 \times 10^{-5} \mathrm{~mol} \mathrm{~L}^{-1}$ & 29 \\
\hline Taxol (anti câncer) & Urina & $5,2 \mu \mathrm{g} \mathrm{L}^{-1}$ & 30 \\
\hline Codeína (analgésico) & Formulações farmacêuticas & $\begin{array}{l}5,0 \times 10^{-6} \mathrm{~mol} \mathrm{~L}^{-1}- \\
10,0 \times 10^{-11} \mathrm{~mol} \mathrm{~L}^{-1}\end{array}$ & $31-33$ \\
\hline Cladribine & Urina e sangue & $75,0 \times 10^{-9} \mathrm{~mol} \mathrm{~L}^{-1}$ & 34 \\
\hline Creatina (aminoácido) & & $6,60 \times 10^{-8} \mathrm{~mol} \mathrm{~L}^{-1}$ & 35 \\
\hline Teofilina (broncodilatador) & 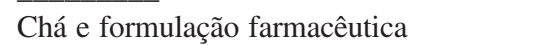 & $0,1 \times 10^{-6} \mathrm{~mol} \mathrm{~L}^{-1}$ & 36 \\
\hline Venlafaxina (antidepressivo) & Formulações farmacêuticas & $0,124 \mathrm{mg} \mathrm{L}^{-1}$ & 37 \\
\hline Prazosina (antihipertensivo) & Formulações farmacêuticas e urina & $7,7 \times 10^{-10} \mathrm{~mol} \mathrm{~L}^{-1}$ & 38 \\
\hline Doxazosina (antihipertensivo) & Formulações farmacêuticas e urina & $2,33 \times 10^{-11} \mathrm{~mol} \mathrm{~L}^{-1}$ & 39,40 \\
\hline Fluoxetina (antidepressivo) & Formulações farmacêuticas & $3,9 \times 10^{-8} \mathrm{~mol} \mathrm{~L}^{-1}$ & 41 \\
\hline Norflaxacin (antibiótico) & Urina & $1,1 \mathrm{mg} \mathrm{ml}^{-1}$ & 28 \\
\hline Timolol (bloqueador beta) & Colírios oftalmológicos & $6,6 \times 10^{-10} \mathrm{~mol} \mathrm{~L}^{-1}$ & 42 \\
\hline 5-Fluorouracil (câncer) & & $7,7 \times 10^{-12} \mathrm{~mol} \mathrm{~L}^{-1}$ & 43 \\
\hline Carbovir 1 (agente anti-HIV) & $\underline{ }$ & $6,0 \times 10^{-5} \mathrm{~mol} \mathrm{~L}^{-1}$ & 44 \\
\hline Viagra & $\overline{\text { comprimidos }}$ & $2,0 \times 10^{-9} \mathrm{~mol} \mathrm{~L}^{-1}$ & 45 \\
\hline Ascorbato & Soro sanguíneo & $2,0 \times 10^{-9} \mathrm{~mol} \mathrm{~L}^{-1}$ & 46 \\
\hline Ácido úrico & Sangue, urina & $\begin{array}{c}1,0 \times 10^{-6} \mathrm{~mol} \mathrm{~L}^{-1}, 1,0 \times 10^{-9} \mathrm{~mol} \mathrm{~L}^{-1} \\
3,0 \times 10^{-7} \mathrm{~mol} \mathrm{~L}^{-1}\end{array}$ & $47-49$ \\
\hline Cisteína & & $1,9 \times 10^{-6} \mathrm{~mol} \mathrm{~L}^{-1}$ & 50 \\
\hline $\begin{array}{l}\text { Xantina e hipoxantina (derivadas } \\
\text { da degradação dos ácidos } \\
\text { nucleotídeos das purinas celulares) }\end{array}$ & $\underline{-}$ & $2,0 \times 10^{-7} \mathrm{~mol} \mathrm{~L}^{-1}$ & 51 \\
\hline Clorexidina (agente antibacteriano) & Formulações farmacêuticas & $0,742 \mathrm{mg} \mathrm{L}^{-1}$ & 52 \\
\hline $\begin{array}{l}\text { PNA (ácido nucléico poliamídico) } \\
\text { e DNA }\end{array}$ & ssDNA e ssPNA & $\begin{array}{l}\text { ssPNA: } 5,0 \mathrm{ng} \mathrm{mL}^{-1} \\
\text { ssDNA: } 50,0 \mathrm{ng} \mathrm{mL}^{-1}\end{array}$ & 53 \\
\hline $\begin{array}{l}\text { N-nitrosomorfolina (carcinogênico - } \\
\text { produzido pela combustão do motor } \\
\text { a diesel) }\end{array}$ & $\begin{array}{l}\text { Monitoramento da presença de } \\
\text { N-nitrosomorfolina em gases produzidos } \\
\text { na combustão de motores a diesel }\end{array}$ & $0,03 \mu \mathrm{g} \mathrm{mL}^{-1}$ & 54 \\
\hline Cafeína & $\begin{array}{l}\text { Chá, café, café descafeinado e } \\
\text { refrigerantes de cola }\end{array}$ & $2,0 \times 10^{-6} \mathrm{~mol} \mathrm{~L}^{-1}$ & 55 \\
\hline Micropartículas de cocaína & 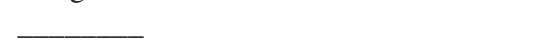 & Detecção qualitativa & 56 \\
\hline $\begin{array}{l}\text { Hipoxantina (derivada da degradação } \\
\text { dos ácidos nucleotídeos das purinas } \\
\text { celulares) }\end{array}$ & $\overline{\text { Grau de deterioração de peixes }}$ & $0,75 \times 10^{-6} \mathrm{~mol} \mathrm{~L}^{-1}$ & 57 \\
\hline Arbutina (propriedades clareadoras) & Produtos cosméticos clareadores & $0,18 \times 10^{-6} \mathrm{~mol} \mathrm{~L}^{-1}$ & 58 \\
\hline Ácido cójico (propriedades clareadoras) & Produtos cosméticos clareadores & $0,17 \times 10^{-6} \mathrm{~mol} \mathrm{~L}^{-1}$ & 59 \\
\hline $\begin{array}{l}\text { Ácido aconítico (acidulante, } \\
\text { intermediário de síntese orgânica de } \\
\text { surfactantes e plastificantes) }\end{array}$ & $\underline{-}$ & $0,184 \mu \mathrm{g} \mathrm{mL}{ }^{-1}$ & 60 \\
\hline Ferro & Azeite de oliva & $0,55 \mu \mathrm{g} \mathrm{L} \mathrm{L}^{-1}$ & 61 \\
\hline Antioxidante & Feijão & - & 62 \\
\hline Vitamina C & Suco de frutas & $0,38 \times 10^{-6} \mathrm{~mol} \mathrm{~L}^{-1}$ & 63 \\
\hline
\end{tabular}


Tabela 1. continuação

\begin{tabular}{|c|c|c|c|}
\hline Composto & Matriz & LD & Ref. \\
\hline TNT & Águas do mar, águas naturais & $25,0 \mu \mathrm{g} \mathrm{L}^{-1}, 200,0-50,0 \mu \mathrm{g} \mathrm{L}^{-1}$ & $64-66$ \\
\hline $\mathrm{Pb}$ & $\begin{array}{l}\text { Amostras certificadas, sedimentos } \\
\text { de rios }\end{array}$ & $\begin{array}{c}<1,0 \mu \mathrm{g} \mathrm{L}^{-1}, 3,0 \times 10^{-6} \mathrm{~mol} \mathrm{~L}^{-1}, \\
0,81 \mu \mathrm{g} \mathrm{L}{ }^{-1}\end{array}$ & $67-69$ \\
\hline Cobre & $\begin{array}{l}\text { Determinação de cobre em sais de } \\
\text { grau analítico }\end{array}$ & $0,05 \mathrm{ng} \mathrm{mL}^{-1}$ & 70 \\
\hline Cianeto & - & $1,2 \times 10-6 \mathrm{~mol} \mathrm{~L}^{-1}$ & 71 \\
\hline Molibdênio & 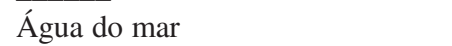 & $0,1 \mu \mathrm{g} \mathrm{L}^{-1}$ & 72 \\
\hline $\mathrm{Cl}-, \mathrm{I}-, \mathrm{Br}-$ & Água mineral & $\begin{array}{l}7,0 \times 10^{-8} \mathrm{~mol} \mathrm{~L}^{-1}\left(\mathrm{Br}^{-} \mathrm{I}^{-}\right) \mathrm{e} \\
2,0 \times 10^{-7} \mathrm{~mol} \mathrm{~L}^{-1} \mathrm{Cl}^{-}\end{array}$ & 73 \\
\hline Prata & Solo & $0,1 \times 10^{-6} \mathrm{~mol} \mathrm{~L}^{-1}$ & 74 \\
\hline Cloreto & Águas naturais & $6,8 \times 10^{-6} \mathrm{~mol} \mathrm{~L}^{-1}$ & 75 \\
\hline $\mathrm{Se}(\mathrm{IV})$ & Meio aquoso & $8,0 \times 10^{-10} \mathrm{~mol} \mathrm{~L}^{-1}$ & 76 \\
\hline $\operatorname{Co}(\mathrm{II})$ & Vidro derretido & - & 77 \\
\hline Enxofre e sulfeto de hidrogênio & Petróleo e seus destilados & $\mathrm{H}_{2} \mathrm{~S}: 10,0 \mathrm{ng} \mathrm{g}^{-1}$ & 78 \\
\hline Thiram (fungicida) & Formulações comerciais & $9,2 \mathrm{mg} \mathrm{L}^{-1}$ & 79,80 \\
\hline Picloram & Águas naturais & $11,0-39,0 \mu \mathrm{g} \mathrm{L}^{-1}$ & 81 \\
\hline Parathion (inseticida) & Águas de rio & $50,0 \times 10^{-9} \mathrm{~mol} \mathrm{~L}^{-1}$ & 82 \\
\hline Bentazon (herbicida) & Formulações comerciais & $10^{-5} \mathrm{~mol} \mathrm{~L}^{-1}$ & 83 \\
\hline Amitrole (herbicida) & Águas de rio & $0,38 \times 10^{-6} \mathrm{~mol} \mathrm{~L}^{-1}$ & 84 \\
\hline Imidacloprid (inseticida) & Águas de rio & $1,0 \times 10^{-8} \mathrm{~mol} \mathrm{~L}^{-1}$ & 85 \\
\hline Nitralin & Águas subterrâneas & $8,7 \times 10^{-11} \mathrm{~mol} \mathrm{~L}^{-1}$ & 86 \\
\hline $\begin{array}{l}\text { Oxadiazon (utilizado em plantações } \\
\text { de arroz) }\end{array}$ & Formulações comerciais & $1,0 \times 10^{-4} \mathrm{~mol} \mathrm{~L}^{-1}$ & 87 \\
\hline Propanil & Formulações comerciais & - & 88 \\
\hline
\end{tabular}

\section{Diclorvos}

O diclorvos (2,2-diclorovinil-dimetil-fosfato) é um pesticida pertencente à classe dos organofosforados, muito utilizado em formulações de inseticidas domésticos e também para a erradicação de insetos voadores e rasteiros. Na agricultura é usado em culturas de batata e hortaliças em geral. Não é muito persistente quando aplicado no solo, mas é altamente tóxico, pois atua sobre o sistema nervoso como inibidor da enzima colinesterase, responsável pela transmissão dos impulsos nervosos.

O diclorvos apresentou eletroatividade sobre o eletrodo gotejante de mercúrio em meio de $\mathrm{Na}_{2} \mathrm{SO}_{4} 0,5 \mathrm{~mol} \mathrm{~L}^{-1}$, o que possibilitou o desenvolvimento de metodologia de análise utilizando a SWV. A otimização dos parâmetros experimentais mostrou que melhor resposta eletroquímica é obtida em $\mathrm{pH} 5 ; f=100 \mathrm{~s}^{-1}$, a $=50 \mathrm{mV}$, $\Delta \mathrm{E}_{\mathrm{s}}=2 \mathrm{mV}$. Nestas condições, foi possível observar a presença de dois picos de redução para o diclorvos em $-0,1 \mathrm{~V}$ e em $-1,1 \mathrm{~V} v s$ $\mathrm{Ag} / \mathrm{AgCl}^{94}$. Após a otimização das condições experimentais, uma curva de trabalho foi construída e a metodologia foi, então, utilizada para análise em águas de córregos da região de São Carlos (córregos Gregório e Monjolinho). Todo tratamento matemático e estatístico ${ }^{90}$ foi realizado no pico de potencial mais negativo.

O Limite de Detecção (LD) foi determinado de acordo com os critérios definidos pela IUPAC ${ }^{91,92}$ e um resumo dos resultados obtidos, tanto para água Milli-Q como para a água dos córregos pode ser visualizado na Tabela 2. Observa-se uma diminuição na inclinação da curva de trabalho, com um conseqüente aumento nos valores dos limites de detecção. Isto foi associado à crescente quantidade de poluentes (principalmente de substâncias orgânicas) contidos nas amostras naturais. No entanto, todos os valores de LD calculados estão abaixo do limite máximo de diclorvos permitido pelo CONAMA - Conselho Nacional do Meio Ambiente $\left(100 \mu \mathrm{g} \mathrm{L}^{-1}\right)^{93}$. Curvas de recuperação foram realizadas e os resultados obtidos, foram satisfatórios ${ }^{94}$.
Tabela 2. Resultados de regressão linear das curvas analíticas do diclorvos, obtidas para as águas Milli-Q e naturais, utilizando HMDE e SWV

\begin{tabular}{lcccc}
\hline Água & $\mathrm{b}\left(\mathrm{A} / \mathrm{mol} \mathrm{L}^{-1}\right)$ & $\mathrm{r}$ & $\mathrm{S}_{\mathrm{B}}(\mathrm{nA})$ & $\mathrm{LD}\left(\mu \mathrm{g} \mathrm{L}^{-1}\right)$ \\
\hline Milli-Q & 5,07 & 0,9980 & 16,9 & 2,2 \\
Gregório & 3,29 & 0,9980 & 32,92 & 5,5 \\
Monjolinho & 2,565 & 0,9968 & 21,37 & 6,6 \\
\hline
\end{tabular}

$\mathrm{b}=$ tangente da curva de trabalho; $\mathrm{r}=$ coeficiente de correlação; $\mathrm{S}_{\mathrm{B}}=$ desvio padrão das medidas do branco e $\mathrm{LD}=$ limite de detecção.

\section{Pentaclorofenol}

Outro trabalho realizado no GMEME foi o desenvolvimento de metodologia para a determinação eletroanalítica do fungicida pentaclorofenol (PCP), utilizando eletrodo de diamante dopado com boro (DDB) ${ }^{95}$. O PCP é um fungicida largamente utilizado como conservante para madeiras, embora seu uso seja proibido em vários países $^{96}$.

Após a otimização dos parâmetros da SWV, como a frequiência $\left(f=100 \mathrm{~s}^{-1}\right)$, amplitude do pulso $(a=50 \mathrm{mV})$ e incremento de varredura $\left(\Delta E_{s}=2 \mathrm{mV}\right)$, observou-se que o PCP sobre o eletrodo de DDB apresenta um único pico de oxidação em torno de $0,80 \mathrm{~V} v s \mathrm{Ag} /$ $\mathrm{AgCl}$, com características irreversíveis e de processo controlado pela adsorção das espécies à superfície eletródica. As curvas analíticas foram obtidas no intervalo de concentração de 0,1 a $6,0 \times 10^{-5} \mathrm{~mol} \mathrm{~L}^{-1} \mathrm{e}$ pode-se determinar o LD para o PCP em água Milli-Q, que apresentou o valor de $2,0 \times 10^{-8} \mathrm{~mol} \mathrm{~L}^{-1}\left(5,5 \mu \mathrm{g} \mathrm{L}^{-1}\right)$. A reprodutibilidade do método foi determinada por medidas sucessivas de cinco soluções diferentes de PCP na concentração de $5,0 \times 10^{-5} \mathrm{~mol} \mathrm{~L}^{-1}$, o coeficiente de variação foi de $1,8 \%$ e, para a análise da repetibilidade, o coeficiente de variação foi de $1,3 \%(n=5)^{95}$. 
Para o estudo do efeito de interferentes foram realizadas medidas com águas coletadas em três pontos diferentes do córrego Monjolinho, de São Carlos, sendo o ponto 1 antes do córrego passar pela cidade (próximo à nascente), ponto 2 no centro da cidade (onde o córrego recebe vários tipos de contaminações) e ponto 3 após cruzar toda a cidade (recebendo todo o tipo de dejetos). Estas análises foram realizadas com o eletrodo de DDB e com eletrodo de gota suspensa de mercúrio (HMDE), com o objetivo de se comparar o efeito dos interferentes nas duas superfícies. Um resumo dos resultados obtidos com eletrodo de DDB encontra-se na Tabela 3, onde se observa que o aumento de impurezas nas amostras causou um aumento no limite de detecção. Isto ocorreu, provavelmente, em função do aumento do conteúdo de matéria orgânica que pode se adsorver na superfície do eletrodo, ou ainda, o próprio pesticida pode reagir com estes contaminantes, diminuindo a sensibilidade do método de determinação.

Tabela 3. Resultados de regressão linear das curvas analíticas do pentaclorofenol, obtidos para as águas Milli-Q e naturais, utilizando o DDB

\begin{tabular}{lclcc}
\hline Água & $\mathrm{r}$ & $\mathrm{S}_{\mathrm{B}}(\mu \mathrm{A})$ & $\mathrm{b}\left(\mathrm{A} / \mathrm{mol} \mathrm{L}^{-1}\right)$ & $\mathrm{LD}\left(\mu \mathrm{g} \mathrm{L}^{-1}\right)$ \\
\hline Milli-Q & 0,9996 & 0,0083 & 1,200 & 5,5 \\
Ponto 1 & 0,9992 & 0,009 & 1,002 & 7,2 \\
Ponto 2 & 0,9990 & 0,015 & 1,004 & 12,0 \\
Ponto 3 & 0,9993 & 0,015 & 0,771 & 15,5 \\
\hline
\end{tabular}

Um estudo similar foi realizado utilizando o $\mathrm{HMDE}^{97}$, no qual foi observado um único pico de redução em torno de $-0,78 \mathrm{~V}$ vs $\mathrm{Ag}$ / $\mathrm{AgCl}$ em tampão fosfato $0,1 \mathrm{~mol} \mathrm{~L}^{-1} \mathrm{e} \mathrm{pH} 9,0$. As curvas analíticas foram obtidas no intervalo de concentração de 0,5 a $8,0 \times 10^{-6} \mathrm{~mol} \mathrm{~L}^{-1}$ para as diferentes amostras de água. Os valores obtidos para o LD, juntamente com os resultados das regressões lineares, são apresentados na Tabela 4. Como era de se esperar, ocorreu um aumento no valor do limite de detecção, de acordo com o conteúdo de matéria de orgânica (Tabela 4). Neste caso, a influência dos poluentes presentes nas amostras ambientais é mais relevante quando comparadas com eletrodo de diamante dopado com boro.

Tabela 4. Resultados de regressão linear das curvas analíticas do pentaclorofenol, obtidos para as águas Milli-Q e naturais, utilizando o eletrodo de mercúrio

\begin{tabular}{lcccc}
\hline Água & $\mathrm{r}$ & $\mathrm{S}_{\mathrm{B}}(\mu \mathrm{A})$ & $\mathrm{b}\left(\mathrm{A} / \mathrm{mol} \mathrm{L}^{-1}\right)$ & $\mathrm{LD}\left(\mu \mathrm{g} \mathrm{L}^{-1}\right)$ \\
\hline Milli-Q & 0,9992 & 0,165 & 0,0341 & 4,0 \\
Ponto 1 & 0,9989 & 0,250 & 0,0300 & 6,6 \\
Ponto 2 & 0,9976 & 0,500 & 0,0288 & 14,0 \\
Ponto 3 & 0,9961 & 0,650 & 0,0285 & 18,0 \\
\hline
\end{tabular}

Após a obtenção das curvas analíticas foram realizados os testes de recuperação utilizando-se os eletrodos de DDB e HMDE (Tabela 5) e verificou-se que o eletrodo de diamante possui uma porcentagem de recuperação em torno de $100 \%$, com uma pequena diminuição em águas contaminadas. Observa-se, ainda, que para o HMDE, mesmo em água Milli-Q, a porcentagem de recuperação é inferior ao eletrodo de diamante.

Os resultados obtidos demonstram que a SWV e o eletrodo DDB são uma excelente alternativa para a determinação de PCP em águas Milli-Q e naturais. Estes resultados são equivalentes aos obtidos com o tradicional HMDE, com algumas vantagens quando se trata de matrizes ambientais contaminadas. No entanto, para os dois siste-
Tabela 5. Resultados das curvas de recuperação para o PCP, obtidos em amostras de águas Milli-Q e naturais, utilizando o eletrodo de DDB e o eletrodo de mercúrio

\begin{tabular}{lclc}
\hline \multicolumn{2}{c}{ DDB } & \multicolumn{2}{c}{ HMDE } \\
Amostra & $\%$ recuperação & Amostra & \% recuperação \\
de água & 100,0 & Milli-Q & 96,0 \\
\hline Milli-Q & 98,0 & Ponto 1 & 95,0 \\
Ponto 1 & 98,0 & Ponto 2 & 93,0 \\
Ponto 2 & 97,0 & Ponto 3 & 92,0 \\
Ponto 3 & & & \\
\hline
\end{tabular}

mas os limites de detecção encontrados $\left(\mathrm{LD}=5,5 \mathrm{e} 4,0 \mu \mathrm{g} \mathrm{L}^{-1}\right.$ para o DBD e o HMDE, respectivamente) estão dentro do limite permitido de PCP em águas pelo Conselho Nacional do Meio Ambiente CONAMA $\left(\mathrm{LD}=10,0 \mu \mathrm{g} \mathrm{L}^{-1}\right){ }^{93}$.

\section{4-clorofenol}

O 4-clorofenol (4-CP) faz parte da família dos clorofenóis, que representa para o ambiente um grave problema de poluição tanto para sistemas aquáticos como terrestres. O 4-CP é um precursor de pesticida e biocida, sendo utilizado nas mais diversas atividades industriais, incluindo o branqueamento da polpa da celulose na indústria de papel, estando presente em resíduos de gás e óleos industriais. $\mathrm{O}$ limite permitido em águas naturais é de $0,1 \mathrm{mg} \mathrm{L}^{-1}$, de acordo com as resoluções do CONAMA ${ }^{93}$.

Os resultados obtidos por SWV, em meio de tampão BR (pH $6,0)$ mostraram a presença de apenas um pico de oxidação para o 4$\mathrm{CP}$ em $0,8 \mathrm{~V}$ vs $\mathrm{Ag} / \mathrm{AgCl}$, com características irreversíveis e de processo controlado pela adsorção das espécies na superfície do eletrodo. Após a otimização do sistema, foram obtidas as curvas analíticas com freqüência de onda quadrada de $100 \mathrm{~s}^{-1}$, amplitude de onda quadrada de $50 \mathrm{mV}$ e incremento de varredura de $2 \mathrm{mV}^{98}$.

O limite de detecção obtido foi $6,4 \mu \mathrm{g} \mathrm{L}^{-1} \mathrm{em}$ água Milli-Q e apresentou os valores de 16,2; 17,0 e 21,5 $\mu \mathrm{g} \mathrm{L}^{-1}$, de acordo com o grau contaminação das águas coletadas em diferentes pontos de São Carlos. Os testes de recuperação mostraram uma eficiência em torno de $100 \%$.

Os resultados obtidos por SWV, em água Milli-Q, foram equivalentes àqueles obtidos por HPLC (Tabela 6). Desta forma, os resultados obtidos indicam que o eletrodo de diamante e a SWV podem ser uma excelente alternativa para análise de 4-CP em águas.

Tabela 6. Resultados de regressão linear das curvas analíticas e valores de limite de detecção e recuperação obtidos para o 4-CF em água Milli-Q, utilizando SWV e HPLC

\begin{tabular}{ccc}
\hline Parâmetro & SWV & HPLC \\
\hline $\mathrm{r}$ & 0,9994 & 0,9998 \\
$\mathrm{~S}_{\mathrm{B}}$ & 0,006 & 0,442 \\
$\mathrm{~b}$ & 0,360 & $1,45 \times 10^{9}$ \\
$\mathrm{LD} /\left(\mu \mathrm{g} \mathrm{L}{ }^{-1}\right)$ & 6,40 & 0,01 \\
$\%$ Recuperação & 100,5 & 96,0 \\
\hline
\end{tabular}

\section{Paraquat}

O uso de ultramicroeletrodos (UME) aliado à SWV também tem sido utilizado, com sucesso no GMEME, para estudos envolvendo o desenvolvimento de metodologias de análises de pesticidas em águas. A utilização de ultramicroeletrodos promove um aumento considerável na qualidade dos dados experimentais como, por exemplo, aumento da resolução, da densidade de corrente e da diminuição dos efeitos da resistência da solução (queda ôhmica) ${ }^{99}$. 
Em função das vantagens inerentes ao uso da SWV e de UMEs, foi desenvolvida uma metodologia eletroanalítica para determinação do herbicida paraquat (1,1'-dimetil-4,4'-bipiridina-dicloreto) em UMEs de Pt e Au com $25 \mu \mathrm{m}$ de diâmetro e fibra de carbono com 7,0 $\mu \mathrm{m}$ de diâmetro ${ }^{100}$.

Em meio de $\mathrm{Na}_{2} \mathrm{SO}_{4} 0,1 \mathrm{~mol} \mathrm{~L}^{-1}$, observou-se a presença de dois picos de redução para o paraquat, em $-0,65 \mathrm{~V}$ (pico 1) e em $-0,98 \mathrm{~V} v \mathrm{~s}$ $\mathrm{Ag} / \mathrm{AgCl}$ (pico 2), onde os valores para os potenciais de pico são independentes da superfície eletródica. Os parâmetros da SWV foram otimizados, permitindo trabalhar com valores de frequiência de aplicação de pulsos de potencial tão elevados como $1000 \mathrm{~s}^{-1}$, o que melhora significativamente a sensibilidade da metodologia. Foi encontrado que este valor bastante elevado de freqüência de aplicação de pulsos de potencial só é possível graças à utilização de UMEs. Este fato está, provavelmente, ligado à difusão mais eficiente dos reagentes à superfície do eletrodo, possibilitando uma rápida renovação da camada de pesticida adsorvida na superfície, com significativa melhora na sensibilidade, o que não ocorre com a utilização de eletrodos convencionais que apresentam uma camada de difusão linear.

Após a otimização das condições experimentais e voltamétricas, onde os valores otimizados foram $f=1000 \mathrm{~s}^{-1}, \mathrm{a}=50 \mathrm{mV}, \Delta \mathrm{E}_{\mathrm{s}}=$ $2,0 \mathrm{mV}, \mathrm{pH}=5,0$, curvas de trabalho lineares foram construídas no intervalo de concentração de $3,0 \times 10^{-6}$ a $1,6 \times 10^{-4} \mathrm{~mol} \mathrm{~L}^{-1}$. Um resumo dos resultados obtidos sobre os três UMEs é apresentado na Tabela 7. Os valores encontrados para os limites de detecção obtidos com a utilização de UMEs, quando comparados a dados já existentes na literatura ${ }^{101,102}$, mostraram-se muito adequados, considerando a utilização desta metodologia na análise de águas. Além disto, os limites obtidos para água Milli-Q estão dentro da faixa necessária para a determinação de contaminação de água potável que, de acordo com a Agência de Proteção Ambiental Americana (EPA), estabelece, para consumo humano, um limite máximo de $100 \mu \mathrm{g} \mathrm{L}^{-1}(100 \mathrm{ppb})^{103}$.

Tabela 7. Resultados obtidos com a construção das curvas de trabalho para detecção do paraquat em diferentes UMEs com freqüência de $1000 \mathrm{~s}^{-1}$, considerando-se o pico 1

\begin{tabular}{ccccc}
\hline $\mathrm{UME}$ & $\mathrm{S}_{\mathrm{B}}(\mathrm{nA})$ & $\mathrm{b}\left(\mathrm{A} / \mathrm{mol} \mathrm{L}^{-1}\right)$ & $\mathrm{r}$ & $\mathrm{LD}\left(\mu \mathrm{g} \mathrm{L}^{-1}\right)$ \\
\hline $\mathrm{Pt}$ & 0,0168 & $2,810 \times 10^{-3}$ & 0,9952 & 3,9 \\
$\mathrm{Au}$ & 0,0442 & $4,796 \times 10^{-3}$ & 0,9995 & 6,2 \\
$\mathrm{FC}$ & 0,0521 & $1,701 \times 10^{-3}$ & 0,9972 & 20,39 \\
\hline
\end{tabular}

\section{Determinação de fármacos}

\section{Ranitidina}

$\mathrm{O}$ estudo do comportamento voltamétrico da ranitidina (N-(2\{[dimetilamino) metil]-2-furanil $\}$-metiltioetil)-N'-metil-2-nitro-1,1'etenodiamina), RNT, princípio ativo de formulações farmacêuticas utilizadas no tratamento de úlceras gástricas e duodenais, foi realizado em meio aquoso com o eletrodo de gota suspensa de mercúrio, HMDE, aliado às técnicas de voltametria cíclica e $\mathrm{SWV}^{104}$. Os estudos foram realizados em tampão fosfato $\left(0,1 \mathrm{~mol} \mathrm{~L}^{-1}\right)$, em intervalo de $\mathrm{pH}$ de 1,5 a 7,0. Os resultados mostraram que a RNT apresenta duas ondas de redução em valores de pH abaixo de 4,0 e apenas uma onda em $\mathrm{pH}$ acima de 4,0, referentes à redução irreversível do grupo nitro para hidroxilamina.

A utilização da SWV mostrou-se mais rápida e sensível (0,02 A/ mol $\mathrm{L}^{-1}$ ) para a utilização da metodologia eletroanalítica na determinação de ranitidina em formulações farmacêuticas, quando comparada às técnicas polarográficas descritas na literatura. Desta forma, para o desenvolvimento do método analítico, foi utilizada a corrente de pico correspondente à segunda onda de redução da ranitidina
$(-0,80 \mathrm{~V}$ vs $\mathrm{Ag} / \mathrm{AgCl})$ em $\mathrm{pH}=3,0$, usando uma freqüência $(f)$ de $100 \mathrm{~s}^{-1}$, uma amplitude de pulso $(a)$ de $50 \mathrm{mV}$ e um incremento de varredura $(\Delta E)$ de $2 \mathrm{mV}$. A curva de trabalho foi construída nestas

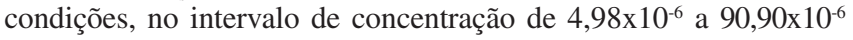
mol L $\mathrm{L}^{-1}$ e o limite de detecção obtido foi $3,5 \times 10^{-8} \mathrm{~mol} \mathrm{~L}^{-1}\left(11 \mu \mathrm{g} \mathrm{L}^{-1}\right)$.

Curvas de recuperação foram realizadas para amostras de formulações farmacêuticas da marca Antak ${ }^{\circledR}$ e medicamento genérico, e as percentagens de recuperação aparente para estas amostras foram de 100,9 e $103,5 \%$, respectivamente.

\section{Dipiridamol}

O dipiridamol, 2,6-bis-(dietanolamina)-4,8-dipiperidinopirimido-[5,4-d]pirimidina (DIP), é utilizado no tratamento de várias doenças cardiovasculares ${ }^{105}$ e suas aplicações recentes como antioxidante em nível biológico ${ }^{106}$ e coativador de compostos anticancerígenos ${ }^{107}$ conferem à molécula substancial importância na área farmacêutica e em medicina.

A redução eletroquímica do DIP em tampão fosfato $0,1 \mathrm{~mol} \mathrm{~L}^{-1}$ e pH 3,0, empregando HMDE e a SWV, mostrou um comportamento voltamétrico irreversível caracterizado por dois picos voltamétricos, em torno de $-0,75 \mathrm{~V}$ e $-1,10 \mathrm{~V}$ vs $\mathrm{Ag} / \mathrm{AgCl}$, correspondendo à perda consecutiva de dois elétrons por etapa ${ }^{108}$.

A justificativa do emprego da SWV neste trabalho dá-se pela sua rapidez e sensibilidade, quando comparada aos trabalhos da literatura utilizando voltametria de redissolução catódica adsortiva ${ }^{109} \mathrm{e}$ polarografia de corrente direta ${ }^{110}$ para a determinação do DIP em formulações farmacêuticas.

Para o desenvolvimento de metodologia analítica para a determinação do DIP em comprimidos e soluções injetáveis de Persantin ${ }^{\circledR}$, vários parâmetros foram otimizados, dentre eles a freqüência $(f=$ $\left.100 \mathrm{~s}^{-1}\right)$, a amplitude do pulso $(a=50 \mathrm{mV})$ e o incremento de varredura $\left(\Delta E_{s}=2 \mathrm{mV}\right)$, com o objetivo de se alcançar maior sensibilidade analítica. Com as condições otimizadas, curvas analíticas foram obtidas no intervalo de 1,28 a $7,02 \times 10^{-6} \mathrm{~mol} \mathrm{~L}^{-1}$. O limite de detecção foi $5,08 \times 10^{-8} \mathrm{~mol} \mathrm{~L}^{-1}(25,64 \mu \mathrm{g} \mathrm{L}-1)$ para o primeiro pico de redução e $1,88 \times 10^{-8} \mathrm{~mol} \mathrm{~L}^{-1}\left(9,49 \mu \mathrm{g} \mathrm{L}^{-1}\right)$ para o segundo pico.

A repetibilidade e a reprodutibilidade da metodologia foram avaliadas para concentrações de padrão na faixa da curva analítica, obtendo-se desvios padrão relativos iguais a $0,97(n=5)$ e $1,15 \%$, respectivamente. Os experimentos de recuperação do DIP foram realizados de acordo com o procedimento descrito por Burns e colaboradores $^{111}$, no intervalo de potencial referente ao segundo pico. A porcentagem média de recuperação foi $100,80 \pm 0,50 \%$ para o comprimido e de 103,36 \pm 0,74\% para a solução injetável. Os resultados obtidos mostraram que a quantidade de medicamento encontrada pelo método da recuperação aparente mostra-se coerente com a informação declarada pelo fabricante e que não há interferência significativa da matriz desses medicamentos na metodologia analítica proposta.

\section{CONCLUSÃO}

A análise dos trabalhos apresentados nesta revisão mostra que a SWV é uma das mais sensíveis técnicas voltamétricas de pulso e pode ser aplicada na determinação de diferentes analitos de interesse. Além disto, quando associada às técnicas de redissolução, pode atingir sensibilidade equivalente às técnicas cromatográficas. Desta forma, a sua aplicação em diferentes estudos realizados no GMEME trouxe uma importante contribuição no desenvolvimento de novas metodologias analíticas para aplicações na detecção de pesticidas em águas poluídas e fármacos.

No entanto, um ponto que ainda necessita de novos estudos quando se fala na SWV como ferramenta no estudo de processos envol- 
vendo mecanismos complexos, tais como EC e ECE, é o aprimoramento e clareza nos critérios de diagnóstico.

Outro fator que vem sendo timidamente estudado é a utilização de multi pulsos de potencial, o que poderá contribuir significativamente no completo estabelecimento da SWV como ferramenta na determinação eletroanalítica de diferentes tipos de compostos.

Com este trabalho é possível observar que a SWV é uma técnica voltamétrica que cresceu significativamente nos últimos anos, e que ainda pode ser mais bem explorada, pois possibilita o alcance de limites de detecção mais baixos do que aqueles obtidos com a utilização de outras técnicas voltamétricas. Assim, esta é uma das motivações para nossa dedicação no desenvolvimento de pesquisas utilizando a SWV.

\section{AGRADECIMENTOS}

Os autores agradecem ao CNPq e à FAPESP pelos auxílios financeiros e bolsas de estudos concedidos, e a R. C. Pires pela ajuda prestada na construção das figuras utilizadas nas Partes 1 e 2 deste trabalho.

\section{REFERÊNCIAS}

1. Osteryoung, J.; Osteryoung, R.; Anal. Chem. 1985, 57, 101A.

2. O'dea, J.; Wojciechowski, M.; Osteryoung, J.; Anal. Chem. 1985, 57, 955.

3. de Souza, D.; Machado, S. A. S.; Avaca, L. A.; Quim. Nova 2003, $26,81$.

4. Lovric, M.; Branica, M.; J. Electroanal. Chem. 1987, 226, 239.

5. Dias-Cruz, M. S.; Esteban, M.; Rodriguez, A. R.; Anal. Chim. Acta 2001, $428,285$.

6. El-Maali, N. A.; El-Hady, D. A.; El-Hamid, M. A.; Seliem, M. M.; Anal. Chim. Acta 2000, 417, 67.

7. Garay, F.; J. Electroanal. Chem. 2001, 505, 100.

8. Garay, F.; Solis, V. M.; J. Electroanal. Chem. 2001, 505, 109

9. El-Maali, N. A.; Wang, J.; Sens. Actuators, B 2000, 76, 211.

10. Mori, V.; Toledo, J. C.; Silva, H. A. S.; Franco, D. W.; Bertotti, M.; J. Electroanal. Chem. 2003, 547, 9.

11. Ivandini, T. A.; Sarada, B. V.; Rao, T. N.; Fujishima A.; Analyst 2003, 128, 924.

12. Masarik, M.; Kizek, R.; Kramer, K. J.; Billova, S.; Brazdova, M.; Vacek, J.; Bailey, M.; Jelen, F.; Howard, J. A.; Anal. Chem. 2003, 75, 2663.

13. Radi, A.; Wahdan, T.; Abd El-Ghany, N.; J. Pharm. Biomed. Anal. 2003, 31,1041 .

14. Zhang, X. H.; Wang, S. F.; Sensors 2003, 3, 61.

15. Ghoneim, M. M.; Tawfik, A.; Khashaba, P. Y.; Anal. Bioanal. Chem. 2003, $375,369$.

16. Zhang, X. H.; Wang, S. F.; Chin. J. Anal. Chem. 2002, 30, 1312.

17. Zhao, H.; Zhang, Y. H.; Yuan, Z. B.; Electroanalysis 2002, 14, 1031.

18. Vela, M. H.; Garcia, M. B. Q.; Montenegro, M. C. B. S. M.; Fresenius ' J. Anal. Chem. 2001, 369, 563.

19. Zen, J. M.; Chen, I. L.; Shih, Y.; Anal. Chim. Acta 1998, 369, 103.

20. de Irazu, S.; Unceta; N.; Sampedro, M. C.; Goicoleta, M. A.; Barrio, R. J.; Analyst 2001, 126, 495

21. Radi, A.; Anal. Lett. 2002, 35, 2449.

22. Msagati, T. A. M.; Ngila, J. C.; Talanta 2002, 58, 605.

23 Radi, A.; Belttagi, A. M.; Ghoneim, H. H.; Talanta 2001, 54, 283.

24. Yilmaz, S.; Uslu, B.; Ozkan, S. A.; Talanta 2001, 54, 351.

25. Uslu, B.; Yilmaz, S.; Ozkan, S. A.; Pharmazie 2001, 56, 629.

26. Radi, A.; El-Sherif, Z.; Talanta 2002, 58, 319.

27. Demircigil, B. T.; Ozkan, S. A.; Coruh, O.; Yilmaz, S.; Electroanalysis 2002, 14, 122 .

28. Oskan, S. A.; Uslu, B.; Anal. Bioanal. Chem. 2002, 372, 582.

29. Uslu, B.; Electroanalysis 2002, 14, 736.

30. Holgado, T. M.; Quintana, M. C.; Pinilla, J. M.; Microchem. J. 2003, 74, 99.

31. Garrido, J. M. P. J.; Delerue-Matos, C.; Borges, F.; Macedo, T. R. A.; Oliveira-Bret, A. M.; Anal. Lett. 2002, 35, 2487.

32. Shih, Y.; Zen, J. M.; Yang, H. H.; J. Pharm. Biomed. Anal. 2002, $29,827$.

33. Zen, J. M.; Chang, M. R.; Chung, H. H.; Shih, Y.; Electroanalysis 1998, $10,536$.

34. de-Los-Santos-Alvarez, N.; Lobo-Castanon, M. J.; Miranda-Ordieres, A. J.; Tunon-Blanco, P.; Electroanalysis 2003, 15, 441.

35. Stojanova, K.; Gulaboski, R.; Mireceski, V.; Petrovska-Jovanovic, S.; Anal. Lett. 1999, 32, 2937.
36. Zen, J. M.; Yu, T. Y.; Shih, Y.; Talanta 1999, 11, 635.

37. Lima, J. L. F. C.; Loo, D. V.; Delerue-Matos, C.; da Silva, A. S. R.; Farmaco 1999, 54, 145.

38. Arranz, A.; Moreda, J. M.; Arranz, J. F.; Chem. Anal. 2000, 19, 31

39. de Betono, S. F.; Garcia, A. A.; Valentin, J. F. A.; J. Pharm. Biomed. Anal. 1999, 20, 621

40. Arranz, A.; Moreda, J. M.; Arranz, J. F.; Mikrochim. Acta 2000, 134, 69.

41. da Silva, A. M. S. R.; Lima, J. C.; Teles, M. T. O.; Brett, A. M. O.; Talanta 1999, 49, 611 .

42. Arranz, A.; Dolara, I.; de Betono S. F.; Moreda, J. M.; Cid, A.; Arranz, J. F.; Anal. Chim. Acta 1999, 389, 225.

43. Mirceski, V.; Gulaboski, R.; Jordanoski, B.; Komorsky-Lovric, S.; J. Electroanal. Chem. 2000, 490, 37.

44. Lemoult, S. C.; Cheng, Y.; Kontturi, K.; Schiffrin, D. J.; Electrochem. Commun. 2000, 2, 244.

45. Berzas, J. J.; Rodriguez, J.; Castañeda, G.; Villaseñor, M. J.; Anal. Chim. Acta 2000, 417, 143.

46. Mo, J. W.; Dgorevc, B.; Anal. Chem. 2001, 73, 1196.

47. Raj, C. R.; Ohsaka, T.; J. Electroanal. Chem. 2003, 540, 69.

48. Zen, J. M.; Jou, J. J.; Ilangovan, G.; Analyst 1998, 123, 1345

49. Zheng, L. Z.; Wu, S. G.; Lin, X. Q.; Nie, L.; Rui, L.; Electroanalysis 2001 13,1351

50. Zen, J. M.; Kumar, A. S.; Chen, J. C.; Anal. Chem. 2001, 73, 1169.

51. Cavalheiro, E. T. G.; Brajter-Toth, A.; J. Pharm. Biomed. Anal. 1998, 19, 217

52. Sarna, K.; Fijalek, Z.; Chem. Anal. 1999, 44, 269.

53. Tomschik, M.; Jelen, F.; Havran, L.; Trnkova, L.; Nielsen, P. E.; Palecek, E.; J. Electroanal. Chem. 1999, 476, 71.

54. Hernandez, L.; Hernandez, P.; Vicente, J.; Blanco, M. H.; Anal. Chim. Acta 1997, 356, 239

55. Zen, J. M.; Ting, Y. S.; Shih, Y.; Analyst 1998, 123, 1145.

56. Komorsky-Lovric, S.; Galic, I.; Penovski, R.; Electroanalysis 1999, 11, 120.

57. Zen, J. M.; Lai, Y. Y.; Ilangovan, G.; Kumar, A. S.; Electroanalysis 2000, 12,280

58. Shih, Y.; Zen, J. M.; Anal. Chim. Acta 2000, 412, 63

59. Shih, Y.; Zen, J. M.; Electroanalysis 1999, 11, 229

60. Spirevska, I.; Soptrajanova, L.; Gulaboski, R.; Anal. Lett. 2000, 33, 919.

61. Diaz, T. G.; Guiberteau, A.; Soto, M. D. L.; Ortiz, J. M.; J. Agric. Food Chem. 2003, 51, 3743 .

62. Perusse, P.; Leech, D.; Electroanalysis 2003, 15, 573.

63. Zen, J. M.; Tsai, D. M.; Yang, H. H.; Electroanalysis 2002, 14, 1597.

64. Wang, J.; Thongngamdee, S.; Anal. Chim. Acta 2003, 485, 139.

65. Wang, J.; Bhada, R. K.; Lu, J. M.; MacDonald, D.; Anal. Chim. Acta 1998, 361,85

66. Wang, J.; Polsky, R.; Tian, B. M.; Charathi, M. P.; Anal. Chem. 2000, 72, 5285

67. Crowley, K.; Cassidy, J.; Electroanalysis 2002, 14, 1077.

68. Saterlay, A. J.; Agra-Gutierrez, C.; Taylor, M. P.; Marken, F.; Compton, R. G.; Electroanalysis 1999, 11, 1083.

69. Zen, J. M.; Chung, H. H.; Kumar. A. S.; Anal. Chim. Acta 2000, 421, 189.

70. Shams, E.; Anal. Lett. 2000, 33, 465.

71. Anh, N. B. H.; Sharp, M.; Anal. Chim. Acta 2000, 405, 145.

72. Sun, Y. C.; Mierzwa, J.; Lan, C. R.; Talanta 2000, 52, 417.

73. Parham, H.; Zargar, B.; Anal. Chim. Acta 2002, 464, 115.

74. Tsai, Y. C.; Coles, B. A.; Holt, K.; Foord, J. S.; Marken, F.; Compton, R. G.; Electroanalysis 2001, 13, 831 .

75. Seymour, E. H.; Lawrence, N. S.; Compton, R. G.; Electroanalysis 2003, 15,689

76. Sladkov, V.; David, F.; Fourest, B.; Anal. Bioanal. Chem. 2003, 375, 300.

77. De Stycker, J.; Westbroek, P.; Temmerman, E.; Electrochem. Commun. 2002, 4, 41 .

78. Kalal, H. S.; Beigi, A. A. M.; Farazmand, M.; Tash, S. A.; Analyst 2000, $125,903$.

79. Zhao, Y. G.; Zheng, X. W.; Huang, Z. Y.; Yang, M. M.; Anal. Chim. Acta 2003, 482, 29

80. Hernandez- Olmos, M. A.; Agui, L.; Yanez-Seno, P.; Pingarron, J. M.; Electrochim. Acta 2000, 46, 289

81. Massaroppi, M. R. C.; Machado, S. A. S.; Avaca, L. A.; J. Braz. Chem. Soc. 2003, 14, 113.

82. Zen, J. M.; Jou, J. J.; Kumar, A. S.; Anal. Chim. Acta 1999, 396, 39.

83. Garrido, E. M.; Lima, J. L. C.; Delerue-Matos, C. M.; Brett, A. M. O.; Talanta 1998, 46, 1131 .

84. Zen, J. M.; Kumar, A. S.; Chang, M. R.; Electrochim. Acta 2000, 45, 1691.

85. Guiberteau, A.; Galeano, T.; Mora, N.; Parrilla, P.; Salinas, F.; Talanta 2001, $53,943$.

86. Arranz, A.; Betoño, F. S.; Moreda, J. M.; Arranz, J. M.; Talanta 1997, 45, 417. 
87. Garrido, E. M.; Lima, J. L. F. C.; Delerue-Matos, C.; Borges, M. F. M. ; Brett, A. M. O.; Electroanalysis 2001, 13, 199.

88. Garrido, E. M.; Lima, J. L. C.; Delerue-Matos, C.; Borges, F.; Silva, A. M. S.; Brett, A. M. O.; Anal. Chim. 2001, 434, 35

89. Reuber, M. D.; J. Toxicol. Environ. Health 1990, 7, 207.

90. Miller, J. C.; Miller, J. N.; Estatística para Química Analítica, AddisonWesley: Iberoamerican S.A.: Wilmington, 1993.

91. Long, G. L.; Winefordner, J. D.; Anal. Chem. 1983, 55, 712.

92. Skoog, D. A.; Holler, F. J.; Nieman, T. A.; Fundamental of Analytical Chemistry, 5ed., Saunders Golden Sunburst Series: Philadelphia, 1998.

93. http://www.aga-ambiental.com.br/leis/resoluo conama 20-86.doc, acessada em Setembro 2002.

94. Avaca, L. A.; Oliveira Junior, R. T. S.; Machado, S. A. S.; 51 International Simposium on Electroanalysis, Warsow, Poland, 2000.

95. Codognoto, L.; Machado, S. A. S.; Avaca, L. A.; Diamond Relat. Mater 2002, 11, 1670

96. Crosby, D. G.; Pure Appl. Chem. 1981, 53, 1051.

97. Codognoto, L.; Machado, S. A. S.; Avaca, L. A. Em Diamond Mater; VII The Electrochem. Soc. Proccedings Series; Swain, G. M.; Davidson, J. L.; Angus, J. C.; Ando, T.; Brown, W. D., eds.; Pennington, 2001, vol. 7, p. 34.
98. Pedrosa, V. A.; Codognoto, L.; Avaca, L. A.; Quim. Nova 2003, 26, 844.

99. Wightman, R. M.; Wipf, D. O. Em Electroanalytical Chemistry (a series of advances); Bard, A. J., ed.; Marcel Dekker: New York, 1984, vol. 14

100. de Souza, D.; Machado, S. A. S.; Quim. Nova 2003, 26, 644.

101. Huebra, M. J. G.; Hernadéz, P.; Ballesteros, Y.; Hernadéz, L.; Talanta 2001, 54, 1077.

102. Fung, Y. S.; Mak, J. L. L.; Electrochim. Acta 1999, 44, 3855.

103. http://www.epa.gov/fedrgstr/EPA-PEST/1997/August/Day-29/p23094.htm, acessada em Junho 2002.

104. Malagutti, A. R.; Mazo, L. H.; J. Braz. Chem. Soc. 2003, 14, 274.

105. Darbar, D.; Gillespie, N.; Main, G.; Bridges, A. B.; Kennedy, N. S. J.; Pringle, T. H.; Mcneill, G. P.; Am. J. Cardiol. 1996, 78, 736.

106. Iuliano, L.; Pedersen, J. Z.; Rotilio, G.; Ferro, D.; Violi, F.; Free Rad. Biol. Med. 1995, 18, 239.

107. Curtin, N. J.; Turner, D. P.; Eur. J. Cancer 1999, 35, 1020.

108. Toledo, R. A.; Castilho, M; Mazo, L. H.; $11^{\circ}$ Encontro Nacional de Ouímica Analítica, Campinas, Brasil, 2001.

109. Zeng, X.; Lin, S.; Hu, N.; Talanta 1993, 40, 1183.

110. Tuncel, M.; Yazan, Y.; Dogrukol, D.; Atkosar, Z.; Anal. Lett. 1991, 24, 1837.

111. Burns, D. T.; Danzer, K.; Townshend, A.; Pure Appl. Chem. 2001, 1, 1. 